\title{
Le forum débat : un dispositif d'apprentissage collaboratif en formation initiale d'enseignants
}

\author{
Gérard Sensevy - Yves Kuster - Françoise Hélary \\ Geneviève Lameul
}

CREAD

IUFM de Bretagne

153, rue de Saint-Malo

F-35043 Rennes cedex

\{gerard.sensevy, yves.kuster, francoise.helary, genevieve.lameul\}@bretagne.iufm.fr

RÉSUMÉ. Dans cette contribution, nous étudions un forum débat situé en formation initiale d'enseignants. Grâce à une analyse à la fois structurale et intrinsèque du contenu des échanges, appuyée sur certaines notions théoriques didactiques, nous montrons la spécificité discursive de cette forme de forum dans ses effets formatifs sur les professeurs en formation. Nous soulignons in fine tout l'intérêt de tels forums, lorsqu'ils sont intégrés au sein de dispositifs qui permettent de faire évoluer les responsabilités respectives du formateur et des formés dans le processus de formation.

ABSTRACT. This article investigates the impact of an online debate (forum debate) in a teacher training programme. This study, based on several concepts used in didactic research, focuses on the impact of the forum debate on teacher trainees' professional development, by analysing its structural features and intrinsic discursive properties. It aims at showing how the integration of such a debate can create the potential for a change in the training process by assigning new roles and cognitive responsibility to both instructors and learners.

MOTS-CLES : formation à distance, forum débat, argumentation distribuée, contrat didactique, dévolution, analyse a priori.

KEYWORDS: open distance learning, forum debate, distributed argumentation, didactic contract, devolution, a priori analysis.

D\&S - 3/2005. L'enseignement scolaire, pages 311 à 330 


\section{Introduction}

Cet article rend compte d'une recherche en cours, menée par une équipe de l'IUFM de Bretagne (CREAD Université Rennes2, IUFM de Bretagne) qui s'efforce d'apporter des éléments de compréhension, en vue d'une théorisation qui reste à établir, d'un dispositif d'apprentissage collaboratif à distance ${ }^{1}$ utilisé dans deux disciplines, l'anglais et les sciences de la vie et de la terre (SVT). L'identification et l'exploration des spécificités communes à ces deux dispositifs doivent permettre à terme de mieux cerner leur impact potentiel en terme de formation professionnelle sur un public de jeunes professeurs en formation initiale.

L'orientation de la problématique spécifique est la suivante : dans la perspective d'une approche didactique, comment utiliser certains concepts, notamment ceux de contrat didactique, de dévolution et de milieu, pour mieux décrire, comprendre et modéliser les formes didactiques inédites produites dans ce type de dispositif ?

Dans la première partie de cette contribution, nous décrivons les caractéristiques du dispositif de formation dans lequel s'insère l'utilisation d'un forum de discussion centré sur un débat d'idées argumenté, objet de notre réflexion dans le présent article, que nous désignerons à présent sous le terme de forum débat.

La deuxième partie de cette contribution est consacrée à la présentation rapide de la méthodologie de recherche: relative au recueil et à l'analyse d'un corpus de données issues des forums débats mis en place dans les deux dispositifs observés.

La troisième partie réservée à l'analyse du corpus proprement dite, est développée en deux sous parties : une rapide analyse structurale du fonctionnement du forum suivie d'une analyse intrinsèque du contenu des échanges.

Nous concluons en montrant dans quelles perspectives ce type de forum débat à distance peut trouver une place probante en formation initiale des professeurs.

\section{Le dispositif étudié : contextualisation et description générale}

Les forums débats dont nous présentons l'analyse sont intégrés dans deux dispositifs de formation ouverte et à distance de l'IUFM de Bretagne mis en place dans les deux disciplines citées, en seconde année de formation initiale de professeurs de lycée et de collège (PLC2). Ces dispositifs ${ }^{2}$ utilisent une plate-forme de formation qui rassemble différentes fonctionnalités de communication (messagerie, espace de mise en commun, espace de discussion, cafétéria...). La mise à disposition de cet outil est destinée à permettre aux professeurs en formation de travailler collaborativement à distance, en se connectant à partir de leur domicile ou

1. Selon la définition de consensus donnée par le Collectif de Chasseneuil (2000).

2. Pour une description plus détaillée, à partir du modèle d'Engeström (1987), cf. Kuster, Hélary, Lameul (2005, à paraître). 
de l'établissement scolaire où ils ont été affectés en tant que professeur stagiaire. Ce choix d'utilisation a été orienté par les recherches antérieures menées à l'IUFM notamment à partir des critères d'utilité, d'utilisabilité, d'acceptabilité (Tricot, 2003).

Les objectifs plus spécifiquement assignés au développement de ces dispositifs sont l'introduction d'une plus grande flexibilité dans la gestion du temps de formation, l'accroissement de l'efficacité de la formation par le partage de ressources et l'augmentation des échanges entre professeurs stagiaires et formateurs, la recherche d'une articulation plus étroite entre la formation dite «théorique » à l'IUFM et les stages pratiques en établissement scolaire. Ces dispositifs visent donc à donner plus de cohérence et de sens à la formation des professeurs stagiaires des deux groupes concernés, représentatifs, du point de vue des critères d'âge et de sexe, de la population totale des PLC2 de l'IUFM de Bretagne.

Différentes activités sont proposées par les formateurs aux professeurs stagiaires pour mettre en place une dynamique collaborative dans ce contexte de formation hybride où s'articulent travail en présence et travail à distance. Les activités proposées vont de la mutualisation de ressources où les professeurs en formation ont une liberté d'initiative importante à des pratiques s'inscrivant dans un cadre institutionnellement plus contraint (accompagnement du mémoire professionnel par exemple), ou plus fortement scénarisé par les concepteurs et animateurs des dispositifs.

Durant l'année scolaire 2004-2005, les professeurs stagiaires d'anglais et de SVT, ont participé sur leurs plates-formes respectives, à un forum débat sur un thème identique: l'interrogation écrite surprise, pratique professionnelle qu'ils rencontrent couramment dans les établissements où ils effectuent leur stage et qu'ils ont connue en tant qu'élèves. Ce type de débat se situe en aval de journées de formation en présentiel consacrées au thème général de l'évaluation des élèves. Il est orienté sur un échange de points de vue et de pratiques de classe. Son objectif est l'élaboration d'une réflexion didactique commune et la co-construction de stratégies d'enseignement permettant éventuellement de dépasser les contradictions initialement exprimées. Cette activité de forum débat est présentée par les formateurs comme «faisant partie intégrante de la formation », et «la participation de tous » est sollicitée. Cependant, s'il est admis que lors de l'entretien de validation le professeur stagiaire puisse être interrogé sur le dispositif de formation à distance, il n'y a pas, pour l'instant, obligation pour lui d'y avoir réellement participé pour être validé. Ces forums sont modérés par le formateur responsable de l'activité qui peut inciter certains stagiaires muets à se manifester, par un rappel discret par mel par exemple, incitation qui se veut encourageante plus que contraignante. 


\section{Méthodologie de l'étude}

\section{Le corpus de données}

Le corpus de données sur lequel nous avons travaillé est constitué de l'intégralité des messages postés sur les deux forums débat ainsi que de quatre entretiens de participants aux forums : deux professeurs stagiaires d'anglais et deux de SVT ont été interrogés quelques mois après la fin du dispositif selon le protocole d'entretien semi-directif suivant: les professeurs stagiaires disposaient, au moment de l'entretien, d'un document écrit où était reproduite la totalité des messages échangés. Ils étaient invités, après un temps de lecture, à s'exprimer sur leur propre contribution au forum, leur perception de l'ensemble de l'argumentation et, plus généralement, sur l'ensemble du dispositif de formation à distance.

\section{Modalités de l'analyse du corpus}

Nous distinguons dans ce qui suit, au sein de l'analyse de notre corpus de données, une analyse de type structural, destinée à repérer quelques dimensions générales des forums, indépendamment des contenus échangés et une analyse de type intrinsèque centrée, elle, sur les contenus mêmes des argumentations et des descriptions de pratiques ; elle s'efforce, à partir du sens de l'action pour les acteurs, tel qu'il a pu être inféré des messages proprement dits et des commentaires sur ces messages produits dans les entretiens, de comprendre la logique pratique ${ }^{3}$ inhérente aux actes de discours produits dans le forum. Cette analyse s'effectue à l'aide de quelques notions théoriques issues notamment des travaux de l'approche comparatiste en didactique et de la didactique des mathématiques (Mercier et al., 2002 ; Sensevy et al., 2005 ; Brousseau, 1998). Nous expliciterons succinctement, au fur et à mesure de leur emploi, les concepts utilisés.

\section{Analyse empirique}

\section{Eléments structuraux}

\section{Forum des professeurs stagiaires de SVT}

Un total de 54 messages a été posté durant les 4 semaines du débat (à partir de mi-novembre). Les professeurs stagiaires de SVT inscrits au débat au nombre de 45 , étaient répartis sur quatre sites de formation. Le nombre de stagiaires participant

3. Nous entendons ce terme au sens de la logique inhérente au sens pratique des acteurs, tel qu'il a été par exemple été décrit par Bourdieu (1980). Il s'agit donc d'une logique dont la rationalité ne peut se comprendre sans la prise en compte des contraintes particulières qui pèsent sur l'action en situation. 
(34) représente $75 \%$ de l'effectif des inscrits. On constate une variation du nombre de messages quotidiens, variation qui va de 0 à 12 messages avec une concentration importante de messages entre le 15 et le 18/11/2004 et entre le 01 et le 03/12/2004.

L'organisation du débat comprend 5 fils de discussion. Chaque fil correspond à des prises de position dans le débat (accompagnées souvent de récits d'expériences) ou à des propositions d'alternatives. A noter que les fils de discussion de la première période (novembre) mélangent des apports de professeurs stagiaires des différents sites alors que ceux de la seconde période (décembre) sont plutôt spécifiques de deux sites seulement.

\section{Forum des professeurs stagiaires d'anglais}

48 messages ont été postés durant seulement 5 jours de débat (fin janvier-début février). Les professeurs stagiaires d'anglais inscrits au débat, au nombre de 19, appartenaient à un seul site de formation. La proportion de stagiaires participant $(18 / 19)$ a été de $95 \%$. Le nombre de messages quotidiens a varié de 1 à 17 messages avec une concentration importante de messages les 30 et 31/01/2005.

L'observation de l'organisation des fils de discussion fait apparaître 7 fils de discussion. Comme dans le forum précédent, chaque fil correspond soit à des prises de position dans le débat, accompagnées souvent de récits d'expériences, soit à des propositions d'alternatives. Un fil de discussion relativement long met en jeu presque exclusivement deux professeurs stagiaires (voir plus loin page 325), figure typique, dans un forum, « d'un débat dans le débat ».

Les données quantitatives et l'organisation des fils de discussion fournissent des indices sur les caractéristiques d'ensemble du fonctionnement du forum. Cependant, la construction du discours argumentatif dans le cadre d'un forum débat ne peut se réduire à la simple utilisation des facilités techniques que propose l'outil. Nous allons montrer, dans ce qui suit, comment on gagne à penser les éléments structuraux présentés dans une perspective distribuée plutôt que linéaire.

\section{Analyse intrinsèque}

Notre analyse va se dérouler en quatre temps :

- la construction de quelques éléments d'analyse a priori d'un forum débat portant sur l'IES ${ }^{4}$. Il s'agit d'élaborer la description d'une logique possible de l'argumentation concernant la thématique de l'IES (pages 316 et 317);

- la description synoptique des argumentations produites dans chacun des deux forums étudiés, en nous appuyant sur l'analyse a priori préalablement effectuée. Ce faisant, nous tentons de montrer ce que la production de ces argumentations doit à la forme forum (pages 317 à 321);

4. IES, désormais en lieu et place d'Interrogation Ecrite Surprise. 
- l'élucidation de quelques éléments propres à définir la spécificité de tels forums de discussion (pages 321 à 327) ;

- la prise en compte de ces forums, non seulement en eux-mêmes, mais en tant que pièces fondamentales au sein d'un dispositif de formation. Nous envisageons alors des modalités possibles de leur utilisation dans une visée directement formative (pages 328 et 329).

\section{A propos de la thématique du forum : l'interrogation écrite surprise}

A notre sens, l'analyse intrinsèque d'un forum débat, en tant qu'analyse du contenu de cette discussion, doit prendre appui sur une analyse a priori, effectuée ici par nous-mêmes, de la thématique. Nous essayons ainsi de transposer à l'analyse des forums une pratique devenue relativement courante en didactique des sciences et des mathématiques (cf. notamment Mercier et Salin, 1987) : rappelons que face à une tâche particulière à laquelle un élève est confronté, il s'agit de se demander comment son activité va être organisée, et, en particulier, de déterminer les connaissances sur lesquelles il va devoir/pouvoir s'appuyer pour accomplir la tâche. Cette analyse $a$ priori constitue ainsi une sorte de pivot sur lequel articuler l'analyse a posteriori des productions effectives.

D'une manière analogue, nous effectuons un premier balisage de la thématique proposée à la réflexion des professeurs en formation, en essayant de dégager a priori la structure conceptuelle possible de l'argumentation. Cette mise au point peut être considérée comme une cartographie préalable, théorique, des éléments que nous nous attendons à trouver dans le débat. Précisons qu'elle n'a pas été portée à la connaissance des stagiaires.

Poser la question de l'interrogation écrite surprise, c'est d'abord poser la question du type de contrat didactique (Brousseau, 1998) instauré dans la classe. Si le contrat didactique, dans la classe, peut se concevoir comme un système d'attentes entre le professeur et les élèves à propos du savoir, le fait que l'IES soit précisément une «surprise », et «trompe les attentes » des élèves, peut constituer une rupture de contrat. Une première manière de concevoir la discussion autour de l'IES, peut donc consister à se poser la question de la rupture de contrat qu'elle actualise, et à se demander en quoi cette rupture de contrat, si elle a lieu, peut être profitable à l'enseignement et à l'apprentissage.

Parallèlement, on peut envisager l'IES dans sa fonction didactique : pourquoi le professeur éprouve-t-il le besoin de faire appel à ce type d'évaluation ? La réponse didactique la plus générique possible pourrait être la suivante: l'apprentissage repose sur le parcours, par l'élève, du temps didactique (Chevallard, 1991; Chevallard et Mercier, 1987 ; Sensevy et al., 2005) relatif à un objet de savoir donné (en fait, à un complexe d'objets). Ce parcours est visible dans la progression suivie dans la classe. Mais il ne saurait s'assimiler à la seule présence effective de l'élève dans cette classe. 
Pour apprendre, l'élève doit étudier, c'est-à-dire prendre la responsabilité de construire un rapport personnel aux objets qui lui sont enseignés : il doit donc se ménager un temps pour l'étude, jour après jour, qui lui permette, de manière cumulative, de construire les connaissances visées par l'enseignement. Dans cette perspective, l'IES est pour le professeur un moyen de vérifier que l'étude a bien lieu. Elle atteste que l'activité de l'élève ne se résume pas à sa présence physique dans la classe, ni même à sa présence intellectuelle, mais qu'elle se déploie en dehors de la classe, dans son temps privé qui devient ainsi pour une part un temps de l'étude. Concevoir de cette manière l'IES fait bien prendre conscience que la fonction décrite ici (fonction d'incitation à l'étude), ne suppose nullement la dimension de «surprise ». Si l'IES était une IE régulière, elle remplirait la même fonction didactique, sans pour cela tomber sous la catégorie de «rupture de contrat » que nous avons précédemment désignée.

Cette première analyse conceptuelle de la question de l'IES montre ainsi, au plan didactique, deux composants principaux, susceptibles d'orienter l'analyse des productions effectives.

Dans l'IES, l'élément de «surprise » doit pouvoir être intégré dans le contrat didactique de la classe, sous peine de rupture du contrat didactique, le professeur pouvant être conçu, dans cette pratique, comme celui qui outrepasse ses droits.

La principale fonction didactique de l'IES semble résider dans l'incitation à l'étude : elle est censée constituer un élément de « dévolution ${ }^{5}$ large », cette dernière expression désignant le processus général par lequel le professeur fait en sorte de rendre l'élève « responsable de son étude ».

\section{Présentation générale de l'argumentation construite dans les forums}

Les deux forums (produits respectivement par les professeurs de SVT et les professeurs d'Anglais) peuvent être redécrits, au plan de leur contenu, d'une manière synoptique. Nous allons donner tour à tour un tableau synoptique de chacun d'entre eux, ensemble qui constituera le matériau de notre étude.

Avec les professeurs stagiaires de SVT

Le schéma ci-dessous (figure 1) permet de situer les points essentiels de l'argumentation dans le forum SVT. On distingue en fait deux rapports principaux à l'IES. Une partie des intervenants se place dans une dynamique favorable à cette pratique (partie droite du schéma), l'autre partie produisant plutôt des arguments défavorables (partie gauche du schéma).

5. La dévolution, nous dit Brousseau (1998), est le processus par lequel le professeur fait en sorte que l'élève se sente responsable, au sens cognitif du terme, de la construction de ses apprentissages dans un environnement (milieu) spécifique. 


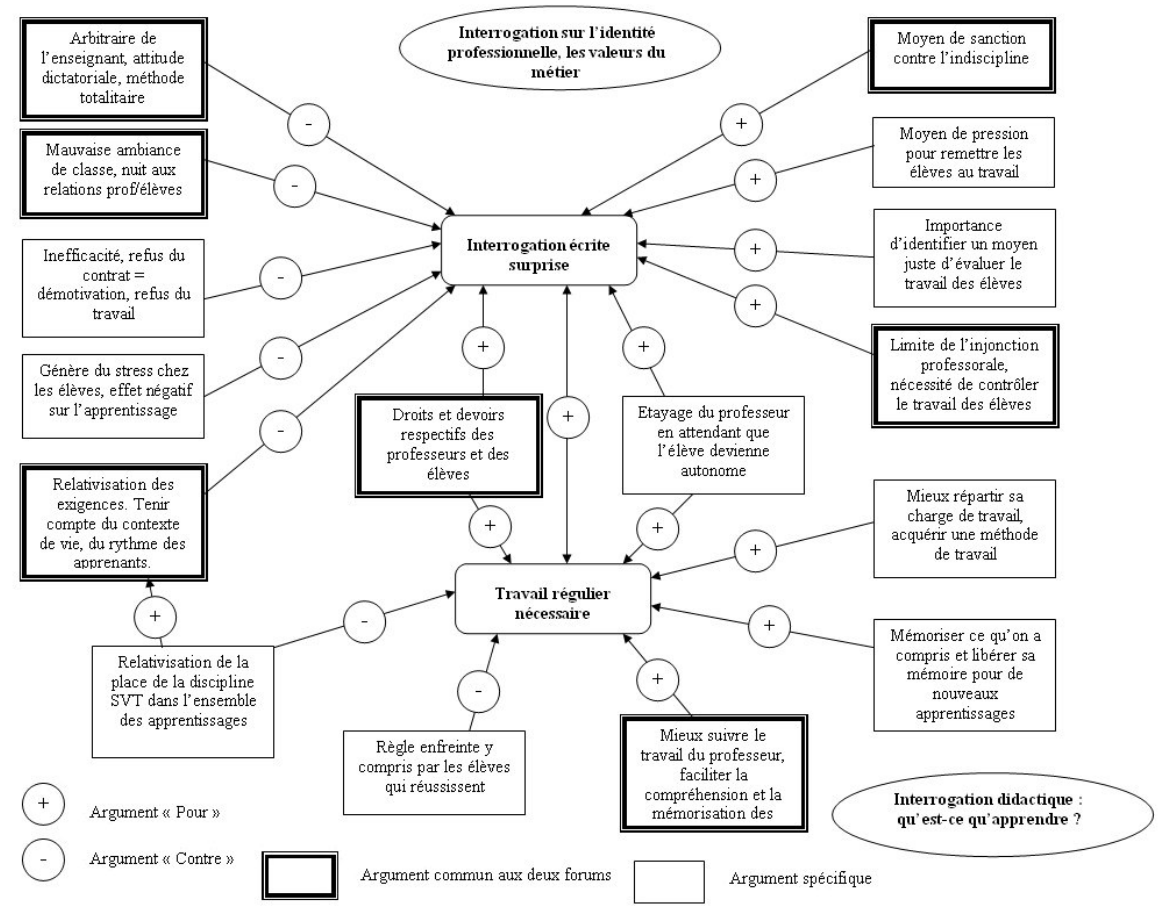

Figure 1. Trame argumentative du débat sur forum (SVT)

Il est intéressant de noter que les argumentations défavorables se fondent quasiment toutes sur l'aspect «surprise » de l'IES, qui apparaît comme une rupture de contrat, et, de ce fait, risque d'introduire dans la classe une «mauvaise ambiance », en compromettant les relations professeur-élèves. Parallèlement, la quasi-totalité des arguments favorables voit dans l'IES un moyen d'initier et de maintenir un travail régulier. L'analyse a priori effectuée au paragraphe précédent se trouve ainsi confirmée : les deux «composantes» argumentatives principales (la nécessité d'intégration de l'IES dans le contrat didactique de la classe ; sa fonction didactique d'incitation à l'étude) se retrouvent déclinées dans les argumentations des participants. Par le jeu des interactions, ceux-ci prennent ainsi conscience à la fois de l'intérêt didactique de l'IES (inciter au travail régulier), et de son inconvénient majeur (la rupture possible, dans la «surprise», du contrat entre élèves et professeurs). On peut trouver ainsi, dans la partie terminale des échanges, des argumentations qui fournissent une sorte de synthèse tout à fait intéressante dans cette perspective, centrées non plus sur la pratique elle-même mais sur la perception qu'ont les professeurs stagiaires des fonctions didactiques que doivent remplir les pratiques d'évaluation en général : 
- la régularité de ces pratiques permet l'incitation à l'étude ;

- cette régularité repose sur une insertion dans le contrat didactique de la classe, et donc dans les attentes et obligations réciproques qui lient professeur et élèves ;

- ces pratiques d'évaluation fonctionnent avant tout comme un encouragement pour les élèves, puisque l'étude régulière permet la réussite presque à coup sûr ;

- l'autonomie de l'élève est encouragée également puisque ces évaluations régulières doivent être conçues par lui comme une sorte de préparation à des évaluations plus vastes (les devoirs proprement dits) pour lesquelles il lui appartient d'organiser son travail, notamment en fonction des résultats obtenus aux interrogations écrites fréquentes et régulières.

Avec les professeurs stagiaires d'Anglais

Le schéma représenté figure 2 permet, d'une façon identique, de situer les points essentiels de l'argumentation dans le forum Anglais.

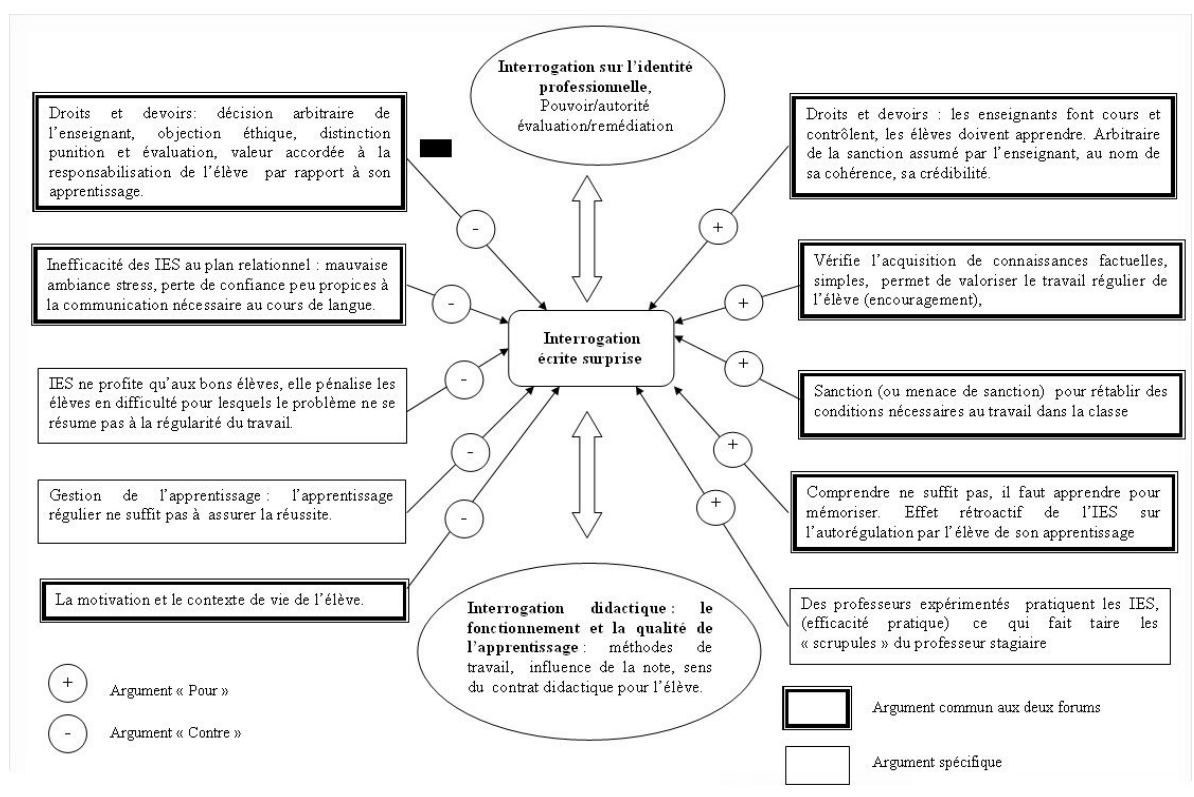

Figure 2. Trame argumentative du débat sur forum (anglais)

De la même façon qu'en SVT, on distingue en fait deux rapports principaux à l'IES, et la parenté des argumentations frappe. Tout se passe comme si la logique pratique, telle qu'elle a pu s'établir dans la réalité effective des interactions didactiques, que nous avons partiellement mise au jour dans notre essai d'analyse $a$ 
priori (cf. plus haut page 316), pesait sur l'argumentation collective, et l'amenait à retenir la nécessité d'étude (intégration d'un travail régulier), tout en reconnaissant les effets indésirables des ruptures de contrat.

L'étude simultanée des deux forums permet par ailleurs de constater l'existence de productions argumentatives qui échappent au moins partiellement à la logique déterminée dans l'analyse a priori.

Tout d'abord, les participants de chacun des forums mettent en évidence le fait que l'IES peut être utilisée (et l'est de fait dans les pratiques des professeurs stagiaires) comme une sanction.

Donner une IES, ce peut être un moyen de pression pour amener les élèves à plus de docilité. L'IES est alors un moyen de maintien de l'ordre. Toutefois, cette pratique de l'IES comme sanction pose problème aux participants, pour des raisons d'éthique professorale, et parce qu'elle risque de compromettre le climat de la classe.

Un autre argument issu des deux forums consiste à considérer l'IES non seulement du point de vue du professeur ou de l'élève dans la matière, mais du point de vue de l'élève confronté à l'ensemble des disciplines. Des voix s'élèvent ainsi dans chacun des forums pour inciter à considérer ce dernier aspect, et à prendre en compte notamment le fait que, à un certain moment du processus d'enseignement, la discipline SVT ou Anglais n'est pas la priorité des élèves (lorsqu'ils ont par exemple un devoir sur table important à préparer dans une autre matière).

Plus généralement, on est frappé par le fait suivant. La dynamique du forum amène peu à peu les intervenants à élargir leurs conceptions, et à intégrer la discussion sur l'IES au sein d'une réflexion didactique et éducative beaucoup plus générale: comment apprend-on? Quel est le rôle de la régularité du travail personnel dans l'étude ? Quels sont les moyens évaluatifs à disposition du professeur pour inciter à l'étude ? Comment concevoir alors l'évaluation dans un perspective formative ? Quel sens peut avoir, au sein d'une évaluation de ce type, l'autonomie de l'élève ? Comment la favoriser ? Comment faire confiance aux élèves, sans tomber dans un angélisme ignorant $\mathrm{du}$ «pouvoir mobilisateur» des notes ?

L'élargissement de ces conceptions porte jusqu'à des interrogations quant aux raisons d'être d'une discipline. Pourquoi, et pour quoi les élèves doivent-ils apprendre ? Quelles sont les limites de l'action de l'enseignant confronté à certains élèves ? Le forum SVT peut ainsi déboucher sur une mise en perspective profonde et bien éloignée de certains cynismes ambiants (C, SVT, 3/12/04) :

"L'idéal serait de n'avoir plus à se soucier de vérifier à chaque cours "s'ils ont appris leur leçon”. Pour cela, il faudrait que le prof arrive à les intéresser (tous!!), à motiver leur curiosité et leur envie d'apprendre à connaître notre planète, tout ce qu'elle héberge de belles choses malgré tout, et à aimer tout ça... (snif..). Alors ils apprendront d'eux même et avec joie! oui oui je sais je sais...c'est sûrement un rêve mais si on ne rêve déjà plus en PLC2. » 
On perçoit dans cette citation comment le problème majeur désigné ici est celui de la dévolution. La « joie » de l'apprentissage, c'est bien celle de l'élève qui trouve dans les savoirs eux-mêmes les raisons d'être de son « existence » en tant qu'élève : la dévolution de l'étude suppose la construction de profits symboliques intimement liés aux savoirs eux-mêmes.

Avant de progresser dans notre étude, mentionnons un dernier point: une caractéristique essentielle, ici, de la discussion produite au sein de ces forums, consiste précisément dans le fait que la totalité factuelle et logique de l'argumentation ne peut se retrouver dans un seul des messages (on voit par exemple comment le message cité ci-dessus n'aborde qu'une dimension seulement - même si elle peut paraître centrale - du problème général collectivement débattu) : l'argumentation se trouve distribuée dans l'ensemble des échanges. C'est un aspect fondamental, sur lequel nous reviendrons

\section{Spécificité du forum débat sur Internet}

Comment penser la spécificité du forum débat tel qu'il a été collectivement produit par les participants?

Le forum débat : un mixte de formes argumentatives

Pour déterminer cette spécificité, il peut être intéressant de caractériser le forum débat à partir de formes déjà existantes. On pourrait ainsi concevoir que ce type de forum débat tient des formes suivantes :

- un écrit argumentatif, dont la dissertation philosophique pourrait constituer le modèle plus ou moins lointain ;

- un témoignage de pratiques, qu'on pourrait voir comme la mise par écrit, par les professeurs, de certaines des descriptions de leurs propres pratiques, du type de celles qu'ils sont amenés à produire, mais la plupart du temps oralement, dans le cadre de l'analyse de pratique exercée à l'IUFM ${ }^{6}$;

- un «débat ou colloque épistolaire », dans la mesure où il est dans la nature même d'un forum qu'un message soit en fait adressé à l'ensemble des participants.

Il faut cependant prendre conscience du fait que le forum ne fonctionne pas, la plupart du temps, comme une juxtaposition de ces différents modèles, mais comme une forme mixte, empruntant à chacune de ces formes, une part de sa spécificité.

Considérons ainsi le message suivant (Ch, SVT, 15/11/04, transcrit ici seulement en partie), produit au début du forum SVT, message riche et assez typique d'un nombre important de messages produits :

6. A l'IUFM de Bretagne, comme dans les autres IUFM de France, les professeurs stagiaires explicitent leurs pratiques de professeur au sein de séminaires d'analyse de pratique, de formes variées, la plupart du temps interdisciplinaire. 
"J'enseigne moi aussi au collège. Il est vrai que le fait d'interroger un élève au début de chaque séance, n'est pas un bien bon stimulant pour le travail personnel régulier... J'ai donc décidé, moi aussi de faire des évaluations de connaissances fréquentes en début de cours. Ils sont prévenus, que la démarche va être régulière... L'objectif pour moi est de les forcer à un travail régulier, et aussi de leur montrer qu'ils comprendront mieux un cours en connaissant bien ce qu'on a vu avant.

Effectivement ce genre de procéder va stresser certains élèves, mais je pense que ceci ne sera pas durable... Quelque part les profs et élèves ont des droits et des devoirs et s'il est du devoir du prof de proposer un cours qui donne envie et qui soit cohérent, il est du devoir de l'élève de s'y pencher personnellement, et au collège le niveau de maturité ne permet pas toujours cette prise de conscience, l'interrogation régulière non plus mais elle en mimera les effets...»

Sans entrer dans la longue étude qu'il mériterait, on peut comprendre comment ce message réfère aux trois formes mentionnées ci-dessus.

Il peut être à un certain moment considéré comme une réflexion philosophique, lorsque l'auteur déclare «Quelque part les profs et élèves ont des droits et des devoirs... mais elle en mimera les effets ».

On perçoit ici comment est affirmée la partition fondamentale des responsabilités et obligations dans le contrat didactique classique : du côté du professeur « un cours qui donne envie et qui soit cohérent» et du côté de l'élève le «devoir» de «s'y pencher personnellement ». Cette dernière formulation (« le devoir... de s’y pencher personnellement »), dans sa simplicité et sa précision, nous semble particulièrement heureuse pour désigner la nécessité de dévolution de l'étude. La concessive finale, relative au «niveau de maturité » qui ne «permet pas toujours [la] prise de conscience » de la nécessité de l'étude, a des accents pascaliens : même si une certaine conscience est absente des comportements des élèves, ces comportements eux-mêmes, en tant que «mîmes » des « effets » (de la prise de conscience), ont une puissance réelle, dont il s'agit de profiter.

Le même message contient clairement un témoignage de pratique : «J'ai donc décidé, moi aussi de faire des évaluations de connaissances fréquentes en début de cours. Ils sont prévenus, que la démarche va être régulière ». Ce témoignage s'épaule mutuellement, pourrait-on dire, avec la réflexion théorisante précédemment mentionnée. Les raisons invoquées (le fait de devoir favoriser la prise de conscience, chez les élèves, de la nécessité de l'étude) et les pratiques évoquées (l'interrogation régulière) semblent former un tout dans l'activité du professeur stagiaire, sans qu'on puisse bien dire si la rationalisation s'est effectuée a posteriori où si elle est à la source de la pratique. Quoi qu'il en soit, on perçoit ici comment la forme forum permet l'inscription de l'auteur dans deux genres discursifs différents (l'argumentation philosophique et le témoignage de pratique), entrelacées plus que juxtaposés, cet entrelacement correspondant probablement à l'entrelacement pratique de la réflexion et de l'action. 
Enfin, on ne saurait rendre raison de l'organisation de ce message si l'on n'appréhendait pas ce qu'il doit à la forme «débat-colloque épistolaire » propre au forum débat. Plusieurs formes témoignent en effet de la polyphonie ${ }^{7}$ foncière de ce message, qui fait systématiquement référence aux messages précédents :

- «J'enseigne moi aussi au collège », le «moi aussi» renvoie au locuteur précédant dans le forum (Cé, SVT, 15/11/04)

- «Il est vrai que le fait d'interroger un élève... », l'expression «il est vrai » fait écho à une argumentation produite également dans le même message précédent (Cé, SVT, 15/11/04)

- «Effectivement ce genre de procédé va stresser certains élèves », l'argument est repris d'un autre message précédent (Y SVT, 15/11/04)

- «Par ailleurs je ne pense pas qu'il s'agisse d'une véritable sanction (facilité à gagner des points)», l'argument est ici opposé à celui d'un troisième message précédent (A, SVT, 15/11/04).

On voit d'ailleurs, sur ce dernier exemple, comment la polyphonie permet ici l'entrelacement «témoignage de pratique » - « argumentation sur les raisons » : c'est pour répondre à la mise en cause de l'IES comme sanction que l'auteur produit la justification «théorique » fondée sur le «partage des responsabilités » entre professeur et élèves.

Ce message, nous avons commencé de le préciser, nous paraît idéal typique d'un certain nombre de messages échangés dans le forum, fonctionnant sur le même type de logique argumentative.

Un tel forum débat peut ainsi se concevoir comme un genre de discours mixte, dans lequel la prise en compte des messages envoyés par les autres participants amène les auteurs à osciller entre des formes elles-mêmes mixtes: témoignages argumentés, arguments empiriquement justifiés - le cours du débat incitant les participants à inscrire leurs productions dans telle ou telle forme. Au delà des deux forums étudiés dans cet article, nous considérons ce type particulier de mixité comme déterminé par la nécessité d'analyse de pratiques propre au forum débat (à distance). Plus largement, on peut conjecturer par ailleurs que la mixité du genre est encore accentuée par certaines spécificités propre de la communication médiatisée par les TIC : sans les possibles et les contraintes proxémiques et non verbales de la communication en présence, cette mixité devient elle-même une ressource argumentative précieuse.

7. Terme employé ici au sens de certains linguistes (notamment Bakhtine (1929), et Ducrot (1984)) et qui consiste à voir dans un énoncé les traces de plusieurs points de vue différents (Charaudeau et Maingueneau, 2002). 
Asynchronie, formes sémiotiques et argumentation

Une autre spécificité du forum débat réside clairement dans les aspects proprement situationnels de la communication qu'il suscite.

L'asynchonie de cette forme permet aux participants d'intervenir dans le différé, ce différé produisant éventuellement une prise de distance conceptuelle. Cette prise de distance est favorisée par la structure sémiotique du forum: l'ensemble des argumentations peut être maintenu devant les yeux, aussi bien dans sa généralité que dans les divers fils de discussion (ce qui est impossible dans le débat oral). Ces propriétés sémiotiques jouent certainement un rôle déterminant dans la polyphonie constatée au sein des messages.

Ces caractéristiques sont à la fois matérielles et logiques: elles situent la communication au sein des forums et déterminent une partie de leur structuration. Notons toutefois que cette détermination n'est pas automatique : l'usage du forum suppose en effet un certain nombre de responsabilités assumées par les participants, sans lesquelles l'efficacité de la forme peut se trouver compromise. C'est ce qu'exprime clairement un des participants au forum SVT (M, SVT, 17/11/04) :

«moi je lis attentivement tous les messages de tout le monde, je note tout, j'essaye de comprendre, sans retenir que la moitié du message, JE CHANGE D'AVIS à la lecture des arguments des autres, puis réécris un message, c'est ça partager ses idées! Et j'aurai attendu des autres les mêmes choses... ».

On voit comment inférer de ce message un «art pratique » du forum débat, qui tient autant aux techniques intellectuelles nécessaires (lire tous les messages, lire chacun intégralement) qu'aux « dispositions d'esprit » qui doivent les accompagner (essayer de comprendre, être capable de changer d'avis à la lecture des messages d'autrui et d'inclure ce changement dans ses messages suivants).

Cet « art pratique », qui repose en grande partie sur la maîtrise de l'outil et de ses usages, n'est pas forcément mis en œuvre par les participants au forum. Les entretiens a posteriori montrent ainsi le coût cognitif de la participation :

"...et puis c'est vrai que devant un écran d'ordinateur, on n'a pas envie de passer des heures à lire quoi. C'est pas un grand confort quand même, ou bien on a un super écran bien confortable mais c'est pas mon cas alors ... pour aller sur le forum il faut quand même passer par le campus, le machin, c'est un peu long l'accès au forum. Si on n'a pas un mel disant qu'on a un message, soit on y a directement mais c'est un peu long le chemin d'accès au forum, ce qui fait que pour les informations, une date, une réunion, çà passait par mel en fait...donc c'est un peu en concurrence pour certaines choses. »(Entretien D, PLC2 SVT, 132).

Dans une telle perspective, on conçoit comment l'asynchronie du forum débat et la pertinence des formes sémiotiques qu'il autorise, supposent de fait un investissement important des participants. Ainsi, les argumentations particulières décrites plus haut illustrent une caractéristique de ce type de forum: les stratégies argumentatives mixtes, dont nous avons pu donner quelques exemples plus haut, 
supposent en fait un travail relativement complexe et intense, dont la nature est tout compte fait relativement inédite dans la pratique des professeurs stagiaires.

Le contrat de communication propre au forum

La fréquentation du forum passe également par le respect de formes propres à ce type de communication. Dans chacun des 2 forums étudiés, nous avons pu relever une sorte d'incident critique, que nous décrivons brièvement.

Dans le forum SVT, l'un des participants intervient beaucoup plus que les autres. S'investissant avec intensité, il est à lui seul responsable, lors des 22 premiers échanges du forum, de plus d'un tiers des signes produits. Les entretiens avec certains des participants au forum montrent que cette attitude n'est pas jugée profitable, puisque ceux-ci se plaignent de la longueur excessive des messages, et de la «monopolisation» du forum par la vision des choses propres à une seule personne.

Dans le forum Anglais, deux participantes, qui par ailleurs se connaissent très bien et se fréquentent en dehors du forum, usent d'un ton familier, faussement polémique, et occupent sur plusieurs messages l'espace de discussion avec ce type d'échanges. Les réactions négatives sont alors exprimées, directement sur le forum.

Ces deux exemples nous font toucher du doigt la prégnance d'un contrat de communication (cf. notamment Bromberg, 2003) propre aux forums de discussion. Comme dans le contrat didactique classique, les normes de la communication n'ont jamais été explicitées dans leur totalité, mais elles n'en sont pas moins agissantes, ainsi que l'explicite Bruillard (2005) : « une sorte de "contrat" entre les membres du groupe, spécifie les conditions pouvant conduire à des formes spécifiques d'interaction ».

On peut attester, ainsi, de l'existence d'un « volume standard » pour les messages sur le forum: trop court, le message ne peut avoir de réelle pertinence (sauf exception, par exemple pour un acquiescement ou une dénégation rapides); trop long, il va « coûter», au plan cognitif, à la fois à l'émetteur et au récepteur.

Les normes de communication tiennent aussi à la nature même du forum, qui se situe dans l'espace public. Toute allusion à une familiarité entre participants peut ainsi rapidement passer pour un private joke qui met à l'écart les non familiers. Dans les entretiens avec les participants, l'accent est mis par plusieurs d'entre eux sur le fait que la discussion en forum trouve une partie de sa pertinence dans son «professionnalisme» : contrairement à la discussion quotidienne, en présence, et même par rapport à la communication par courrier électronique, on y est forcé à la concision, et à la centration sur l'essentiel, c'est-à-dire sur le professionnel, et non sur le personnel.

Il faut ajouter, dans le cas des forums qui nous occupent, que ce «professionnalisme» qui exclut le «personnel» s'établit dans un cadre où l'ensemble des participants (dans le forum SVT, et plus encore dans le forum 
Anglais) se connaît et se fréquente : la partition entre « relations professionnelles » et «relations personnelles » est actualisée, au moins en partie, dans la distinction entre «débat dans le forum» et «discussion de la vie quotidienne». Notons, pour finir sur ce point, que la connaissance mutuelle des participants n'est sans doute pas sans effets sur la nature même des interrelations au sein du forum débat. L'une des deux participantes au forum Anglais impliquées dans la discussion «semi-privée » mentionnée plus haut peut ainsi déclarer (Entretien E, PLC2 Anglais, 87) :

«... c'est ce qu'on disait en groupe entier, c'est pas des choses qu'on ferait sur un forum où on connaît pas les gens. C'est sûr que c'est à adapter ».

Le forum prend place, ainsi, au sein d'un environnement qui contribue à le façonner.

Le forum dans le contrat didactique de la formation

Il est impossible de comprendre finement la nature des échanges au sein des forums étudiés sans les référer à leur situation au sein d'un dispositif de formation. D'une certaine manière, à la différence des forums Internet construits par des «échanges libres », les forums que nous étudions sont des forums «contraints ». En effet, les participants ne peuvent être que clairement conscients du fait que cette modalité de travail est institutionnellement proposée, et que leur participation, sans être tout à fait obligatoire, est «vivement souhaitée ». Par ailleurs, la thématique même du forum, l'IES, est engageante : l'usage des IES, lorsqu'elles sont employées comme «moyen de sanction » (ce qui semble assez souvent le cas), se trouve d'une certaine manière en contradiction avec l'éthique professorale, et les nouveaux règlements intérieurs des établissements, qui préconisent de ne jamais régler des problèmes de disciplines par l'attribution de notes. Les participants sont donc tenus de maintenir une liberté de propos sans laquelle leurs productions seraient vaines, tout en intégrant ces propos dans le contexte institutionnel général de leur formation, et de leur validation.

Les professeurs stagiaires sont donc confrontés à deux types de contraintes particuliers : ils doivent s'exprimer dans le forum, même s'ils n'en n'ont pas vraiment l'inclination; ils savent qu'ils s'expriment en tant que professeurs stagiaires, ce qui signifie que leurs argumentations et leurs pratiques (telles qu'ils en produisent des témoignages) vont être soumises au regard de tous, et en particulier à celui de leurs formateurs. Quelles que soient les assurances que ces derniers peuvent produire - et faire effectivement respecter - quant à la finalité d'analyse de pratique, non prescriptive et non évaluative, du forum débat, les professeurs stagiaires ont à gérer ces contraintes, et la contradiction qui leur est inhérente : parler «librement » de leurs pratiques dans une institution dont la fonction est précisément de les former, c'est-à-dire de déterminer au moins en partie ces pratiques, pour in fine, les évaluer ${ }^{8}$.

8. Même si cette évaluation se produit tout à fait en dehors du cadre du forum débat, qui, nous l'avons précisé, n'est pas évalué en tant que tel. 
L'argumentation distribuée : la production d'un écrit collectif

On est frappé, à la considération du contenu des forums tels qu'ils peuvent être synthétisés dans les tableaux synoptiques figurant plus haut, par le genre de discours spécifique qu'ils constituent. Nous voulons insister sur le fait que la reconstitution de l'argumentation dans de tels tableaux ne produit une catégorie qu'en appui sur une variété de messages se répondant les uns aux autres, en partie de façon redondante.

La place manque ici pour analyser avec précision ce mécanisme, mais il faut intégrer le fait qu'une catégorie donnée, qui devient peu à peu une signification partagée dans le forum, n'apparaît au regard extérieur que dès l'instant où elle se constitue de manière distribuée dans différents messages. Ainsi, une catégorie comme «Relativisation des exigences. Tenir compte du contexte de vie, du rythme des apprenants » (forum SVT), se distribue sur plusieurs messages au sein du forum, certains complétant un message initial centré sur la nécessité de comprendre que «les élèves n'ont pas que les SVT dans la vie», d'autres reprenant, au sein d'une argumentation plus vaste, tout ou partie de cette argumentation comme allant de soi.

Le forum débat apparaît ainsi comme un écrit collectif spécifique, appuyé sur des descriptions de pratiques, dont l'argumentation fondamentalement polyphonique et distribuée, ne se livre que ressaisie par un regard extérieur : celui du participant au forum, dans la mesure où il peut/veut jouer le jeu de cette participation active et créatrice; celui du chercheur, et/ou du formateur qui cherche à tirer profit de la forme forum pour le processus de formation.

Même implicite, la structure de cette argumentation pèse sur les propos des professeurs stagiaires, en produisant peu à peu un réseau de significations partagées, un grounding (Baker et al., 1999), qui finit par constituer un milieu (Brousseau, 1998) : un réseau de contraintes et de ressources argumentatives par rapport auquel les contributions se définissent et sans lequel elles ne sauraient se comprendre.

\section{En matière de conclusion : le forum débat au sein d'un dispositif de formation}

Le forum débat nous est apparu susceptible de produire des effets formatifs avérés ${ }^{9}$ :

- sur les participants au forum eux-mêmes, dans la mesure où il peut présenter les avantages propres à l'écriture professionnelle, tant dans la posture de réflexivité que celle-ci suscite, que dans la pratique du genre «description de pratiques » que ce type d'argumentation appelle quasi nécessairement. La valeur formative de l'analyse de pratiques (notamment le fait qu'elle met à distance le regard évaluatif et qu'elle produit entre les participants une interconnaissance profitable à la fois pour donner à

9. Le corpus limité sur lequel ont porté les analyses nous rend cependant prudents quant à la généralisabilité des résultats obtenus et des conclusions qui suivent. D'autres études, en cours, sont destinées à valider ces résultats et à tester les conjectures produites. 
voir d'autres pratiques que les siennes et pour se persuader que les problèmes qu'on rencontre sont partagés) se trouve ainsi spécifiée dans le forum débat, avec les caractéristiques propres à celui-ci, que nous avons mentionnées plus haut : vertus de l'asynchronie, et de l'espace sémiotique particulier que constitue le forum ;

- sur les formateurs, dans la mesure où les productions des stagiaires prennent le statut de «fenêtres » ouvertes à la fois sur les conceptions et sur les pratiques effectives.

Peuvent ainsi venir dans l'argumentation, motivées en particulier par le jeu de la « dispute », des expressions qui témoignent de rapports à la profession, aux élèves, aux savoirs eux-mêmes, que les formateurs n'avaient pas eu la possibilité de constater. En particulier, un tel forum met en évidence le poids insoupçonné, comme matrice des pratiques mises en œuvre par les jeunes professeurs, de leur propre passé d'élèves, dans le renversement que Bourdieu (1980) avait mis en évidence comme forme majeure de production de dispositions dans le monde social : un professeur se conduit avec ses élèves dans une certaine mesure comme (certains de) ses professeurs (ne) se sont (pas) conduits avec lui lorsqu'il était élève. Les témoignages abondent ainsi, parmi les professeurs stagiaires, de leur propre expérience d'élève, et de pratiques de leurs anciens professeurs qu'ils s'efforcent de (re)mettre à l'ouvrage, ou qu'ils peuvent au contraire considérer comme repoussoirs. Dans une autre perspective, c'est aussi, à travers le discours des stagiaires, à leur propres pratiques que peuvent se trouver confrontés les formateurs: le forum débat peut lors apparaitre comme une occasion d'analyse de la pratique de formation.

Les intérêts formatifs que nous venons de mentionner tiennent à la forme forum débat en elle-même. Mais il paraît décisif, si l'on veut tirer le plus de profit de cette pratique, de la prendre elle-même dans la réflexivité. Dans une telle perspective, les trames argumentatives produites plus haut peuvent jouer un rôle majeur. Leur production elle-même peut être confiée à l'ensemble des participants (aux 2/3 environ du forum, l'un des professeurs stagiaires du forum SVT propose ainsi spontanément, en fichier attaché, aux autres participants, une récapitulation des arguments produits). Dans le forum Anglais, la trame argumentative a été produite collectivement, en présence, par les professeurs stagiaires étudiant l'ensemble des messages échangés. La formatrice animatrice conclut ainsi le forum Anglais, $(03 / 02 / 05)$ :

«Je vous envoie ici en fichier attaché pour faciliter la lecture la totalité des échanges de ce forum débat. Pour qu'il puisse en rester quelque chose, il convient à présent de faire la synthèse des arguments échangés. Pour cela nous nous attacherons à considérer cet ensemble d'échanges comme un tout. Il s'agit d' identifier les différents arguments échangés pour les lier entre eux: classer les arguments, rassembler les arguments qui ont l'air d'aller dans le même sens, éventuellement faire des sous-catégories, inversement les différencier des autres etc., essayer ensuite de discerner les caractéristiques de la progression de l'argumentation.» 
Le contenu de la trame, qui visualise et actualise des significations auparavant distribuées (et donc pas forcément clairement identifiées comme telles) peut jouer le double rôle de mémoire de l'argumentation, et d' «objet argumentatif » destiné à être repris dans une argumentation de type secondaire.

On pourrait par ailleurs imaginer que figure parallèlement à cette trame argumentative un «relevé de descriptions de pratiques», dans lequel les pratiques dont les professeurs stagiaires ont fait témoignage soient consignées, et analysées, au plan de leurs effets conjecturés sur les apprentissages, de leurs avantages et de leurs inconvénients.

Une utilisation formative majeure de tels forums débats pourrait ainsi se concevoir dans le sens d'un déplacement du partage des responsabilités formatives vers les professeurs-stagiaires: les professeurs stagiaires produiraient, grâce à l'entrelacs des descriptions de leurs pratiques effectives et de l'argumentation de leurs raisons d'être, la matière même de la formation ; les formateurs participeraient de façon privilégiée à la clarification conceptuelle des thématiques abordées, et à l'analyse critique des pratiques décrites. Dans une telle perspective, notamment, la distance, à la fois temporelle et spatiale, de la communication médiatisée dans le forum débat, semble alors jouer un rôle décisif : chaque participant peut s'inscrire, à la fois dans ses propres productions (témoignages de pratiques et argumentations quant aux raisons d'être de ces pratiques) et face à celle des autres, dans l'étude et dans la délibération, tout en participant à la construction d'un collectif de pensée élaboré par le travail en retour sur ces productions. L'apport de la distance, dès lors qu'elle s'institue dans le travail formatif, nous apparaît ainsi décisif.

\section{Bibliographie}

Baker, M.J., Hansen, T., Joiner, R., Traum, D., «The role of grounding in collaborative learning tasks », In P. Dillenbourg (Ed.), Collaborative Learning: Cognitive and Computational Approaches, Amsterdam, Pergamon/Elsevier Science, 1999, pp. 31-63.

Bakhtine M., La Poétique de Dostoïevski, Paris, Seuil, 1970.

Bromberg C., Contrat de communication et construction du sens. In J-L Beauvois, (Ed), Rodolphe Ghiglione. Un parcours théorique et son impact, Grenoble, Presses Universitaires de Grenoble, 2001, pp. 33-38.

Bourdieu P., Le sens pratique, Paris, Minuit, 1980.

Bruillard E., «Apprentissage coopératif à distance : quelques repères sur les questions de recherche » (Sous presse), 2005.

Brousseau G., Théorie des situations didactiques, Grenoble, La Pensée Sauvage, 1998.

Charaudeau P., Maingueneau D., Dictionnaire d'analyse du discours, Paris, Seuil, 2002.

Chevallard Y., La transposition didactique, Grenoble, La Pensée Sauvage, 1991. 
Chevallard Y., Mercier, A., Sur la formation historique du temps didactique, Marseille, IREM, 1987.

Collectif de Chasseneuil, Accompagner des formations ouvertes, conférence de consensus, Paris, L'Harmattan, 2000

Ducrot O., Le Dire et le dit, Paris, Minuit, 1984.

Engeström Y., Learning by Expanding: An Activity - Theoretical Approach to Developmental Research». Helsinki: Orienta-Konsultit., 1987.

http://lchc.ucsd.edu/MCA/Paper/Engestrom/expanding/toc.htm

Kuster Y., Hélary F., Lameul G., « Dispositifs de travail collaboratif à distance à l'IUFM de Bretagne en formation initiale », In G-L Baron \& E. Bruillard (Eds), Technologies de communication et formation d'enseignants: vers de nouvelles modalités de professionnalisation? (Sous presse), 2005.

Mercier A., Salin M.-H., «L'analyse a priori, outil pour l'observation », Actes de l'Université d'été "Didactique et formation des maîtres à l'Ecole Elémentaire", Bordeaux, IREM de Bordeaux, 1988.

Mercier, A., Schubauer-Leoni, M.-L., Sensevy, G., (Eds), «Vers une didactique comparée », Revue Française de pédagogie, 141, 5-16, 2002.

Sensevy G., Schubauer-Leoni M.-L., Mercier A., Ligozat F., Perrot G., « An attempt to model the teacher's action in the mathematics class », Educational Studies in Mathematics, 59, 1-2-3, 2005, pp. 153-181.

Tricot A., «Utilité, utilisabilité, acceptabilité : interpréter les relations entre trois dimensions de l'évaluation des EIAH », Colloque EIAH, Strasbourg, 2003. 